\title{
Rifampicin-induced adrenal insufficiency in the acquired immunodeficiency syndrome: difficulties in diagnosis and treatment
}

\author{
Sandra K. Ediger and William L. Isley
}

Department of Medicine, University of Missouri-Kansas City School of Medicine, Kansas City, Missouri 64108, USA.

\begin{abstract}
Summary: Rifampicin, an antituberculous drug, causes increased hepatic metabolism of steroid hormones. We report the case of a patient with the acquired immunodeficiency syndrome treated with rifampicin who had a 'normal' screening test for adrenal insufficiency, yet had clinical evidence of adrenal failure. Diagnostic testing could not be completed due to lack of clinical response to dexamethasone. Both of these findings are due to the unique effects of rifampicin on steroid metabolism.
\end{abstract}

\section{Introduction}

Antituberculous therapy is commonly utilized in patients with the acquired immunodeficiency syndrome (AIDS) for a variety of mycobacterial infections. Rifampicin increases hepatic steroid metabolism $^{1}$ which can lead to increased corticosteroid requirements in patients with treated adrenal insufficiency. ${ }^{2}$ Inadequate adrenal reserve is not uncommon in AIDS patients. ${ }^{3}$ Therefore monitoring AIDS patients treated with rifampicin for signs and symptoms of adrenal insufficiency is essential. We report the case of a patient with AIDS who developed adrenal insufficiency after beginning rifampicin therapy who had a 'normal' screening test for adrenal insufficiency and no response to potent steroid administration, a unique situation due to rifampicin's effects on hepatic steroid metabolism.

\section{Case report}

A 31 year old homosexual male was diagnosed with AIDS after presenting with Pneumocystis carinii pneumonia. Initial therapy with pentamidine was complicated by the development of diabetic ketoacidosis, ultimately resulting in long-term insulin therapy. Several months later, diagnostic workup of persistent diarrhoea revealed acid-fast bacilli on small bowel biopsy, prompting initiation of antituberculous therapy (rifampicin $600 \mathrm{mg}$,

Correspondence: William L. Isley, M.D.

Accepted: 22 December 1987 isoniazid $300 \mathrm{mg}$, and ethambutol $1200 \mathrm{mg}$ daily). Diminishing insulin requirements were noted after the initiation of antituberculous therapy. Approximately 5 weeks after rifampicin therapy was begun, the patient presented to an outside hospital comatose with a serum glucose of $1.3 \mathrm{mmol} / \mathrm{l}$. Prompt clinical improvement followed infusion of dextrose. Upon transfer to the Truman Medical Center examination of the patient revealed blood pressure $70 / 50 \mathrm{mmHg}$ with orthostatic changes, pulse 128 per minute, temperature $36.7^{\circ} \mathrm{C}$, decreased skin turgor, generalized hyperpigmentation, and diffuse abdominal tenderness. Admission laboratory values included serum sodium $117 \mathrm{mmol} / \mathrm{l}$, potassium $5.0 \mathrm{mmol} / 1$, and bicarbonate $14 \mathrm{mmol} / 1$. The patient's clinical status promptly improved with intravenous hydrocortisone therapy. Antituberculous medications which had been discontinued one week earlier were restarted, but the patient remained off insulin therapy.

Because of the suspicion of adrenal insufficiency, a rapid tetracosactrin (Cortrosyn) stimulation test was performed prior to hydrocortisone therapy. Serum cortisol increased from $607 \mathrm{nmol} / 1$ to $635 \mathrm{nmol} / 1$ (normal greater than $500 \mathrm{nmol} / \mathrm{l}$ ). Due to the equivocal tetracosactrin stimulation test in the setting of clinical adrenal insufficiency, hydrocortisone therapy was discontinued and treatment with dexamethasone $3 \mathrm{mg}$ intravenously every 8 hours was begun in order that further adrenal testing could be carried out. However, the patient again became hypotensive and hypoglycaemic, necessitating reinstitution of hydrocortisone therapy

(C) The Fellowship of Postgraduate Medicine, 1988 
(100 mg every 8 hours). Prompt stabilization of the patient's clinical status occurred. A second tetracosactrin stimulation test done during dexamethasone therapy revealed no increase in the baseline serum cortisol of $497 \mathrm{nmol} / \mathrm{l}$.

Subsequent laboratory identification of the acidfast bacilli from the small bowel biopsy revealed Mycobacterium avium-intracellulare. Rifampicin therapy was therefore discontinued. Clinical deterioration subsequently followed with the development of hypertension and volume overload. Steroid doses were reduced, but the patient ultimately expired.

\section{Discussion}

Rifampicin, a semisynthetic derivative of a product of Streptomyces mediterranei, is frequently prescribed for mycobacterial infections. Side effects are uncommon, but can include liver function abnormalities. The effect of rifampicin on hepatic metabolism of glucocorticoids, presumably through induction of microsomal enzymes, has occasionally manifested itself in adrenal insufficiency in treated Addison's disease ${ }^{2}$ or loss of immunosuppression in renal transplant patients. ${ }^{4}$

Two facets of our patient's course are of particular note. The initial tetracosactrin stimulation test would have been interpreted as normal by conventional criteria, ${ }^{5}$ but the high baseline cortisol value with minimal response presumably indicates inadequate adrenal reserve in a patient with 'shunting' of endogenous glucocorticoids through hepatic microsomal pathways due to

\section{References}

1. Edwards, O.M., Courtenay-Evans, R.J., Galley, J.M., Hunter, J. \& Tait, A.D. Changes in cortisol metabolism following rifampicin therapy. Lancet 1974, ii: $549-551$.

2. Kyriazopoulou, V., Parparousi, O. \& Vagenakis, A.G. Rifampicin-induced adrenal crisis in Addisonian patients receiving corticosteroid replacement therapy. $J$ Clin Endocrinol Metab 1984, 59: 1204-1206.

3. Membreno, L., Irony, I., Dere, W., Klein, R., Biglieri, E.G. \& Cobb, E. Adrenocortical function in the acquired immunodeficiency syndrome. $J$ Clin Endocrinol Metab 1987, 65: 482-487.

4. Buffington, G.A., Dominguez, J.H., Piering, W.F., Hebert, L.A., Kauffman, H.M. \& Leman, J. Jr. Interaction of rifampicin and glucocorticoids: adverse effect on renal allograft function. JAMA 1976, 236: 1958-1960. rifampicin therapy. Furthermore, the patient's failure to respond clinically to dexamethasone, a steroid with a glucocorticoid potency ratio of approximately $30,{ }^{6}$ is consistent with the exaggerated effects of rifampicin on dexamethasone metabolism. In normal volunteers treated with rifampicin, Kawai found that cortisol clearance was increased $21 \%$ while dexamethasone clearance was augmented $372 \% .^{7}$

AIDS patients not uncommonly have decreased adrenal reserve. ${ }^{3,8}$ Since mycobacterial infections are common in these patients, care must be exercised in administering rifampicin since adrenal insufficiency may occur. Screening tests for adrenal insufficiency may be equivocal. It is probably wise to use prolonged corticotrophin stimulation tests in these patients to assess adrenal reserve and to have a low threshold to treat such patients. Choice of therapy must be guided by the knowledge that larger than customary doses of corticosteroids are necessary to meet requirements. If dexamethasone is utilized so that adrenal reserve studies may be carried out, extremely large (at least $20-25 \mathrm{mg}$ daily) doses are necessary due to the pronounced effect of rifampicin on dexamethasone metabolism.

\section{Acknowledgments}

The authors gratefully acknowledge Dr Stuart Chen for: translation assistance, the Truman Medical Center Endocrine Laboratory for performance of hormone assays. Jean Sarkis, M.L.S. for assistance in library research, and Ms Stephanie Etheridge for expert secretarial assistance.
5. May, M.E. \& Carey, R.M. Rapid adrenocorticotropic hormone test in practice: retrospective review. Am J Med 1985, 79: 679-684.

6. Terrell, J.B. \& Baxter, J.D., Glucocorticoid therapy. In: Felig, P., Baxter, J.D., Broadus, A.E. \& Frohman, L.A. (eds) Endocrinology and Metabolism (2nd ed.). McGraw-Hill, St. Louis, 1987, pp 788-817.

7. Kawai, S. A comparative study of the accelerated metabolism of cortisol, prednisolone and dexamethasone in patients under rifampicin therapy. Nippon Naibunpi Gakkai Zasshi 1985, 61: 145-161.

8. Greene, L.W., Cole, W., Greene, J.B. et al. Adrenal insufficiency as a complication of the acquired immunodeficiency syndrome. Ann Intern Med 1984, 101: 497-498. 